\title{
Micromixer Based Preparation of Functionalized Liposomes and Targeting Drug Delivery
}

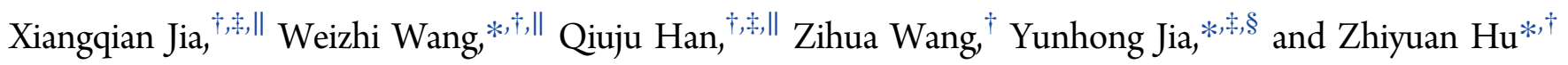 \\ ${ }^{\dagger}$ CAS Key Laboratory for Biomedical Effects of Nanomaterials \& Nanosafety, National Center for Nanoscience and Technology of \\ China, Beijing 100190, China \\ ${ }^{\ddagger}$ Pharmacy College and ${ }^{\S}$ College of Basic Science, Liaoning Medical University, Jinzhou, Liaoning 121001, China
}

Supporting Information

ABSTRACT: We present here a specific targeting nanocarrier system by functionalization of liposomes with one new type of breast cancer targeting peptide (H6, YLFFVFER) by a micromixer with high efficiency. Antitumor drugs could be successfully delivered into human epidermal growth factor receptor 2 (HER2) positive breast cancer cells with high efficiency in both in vivo and ex vivo models.

KEYWORDS: Nanocarrier, HER2, Antitumor, YLFFVFER

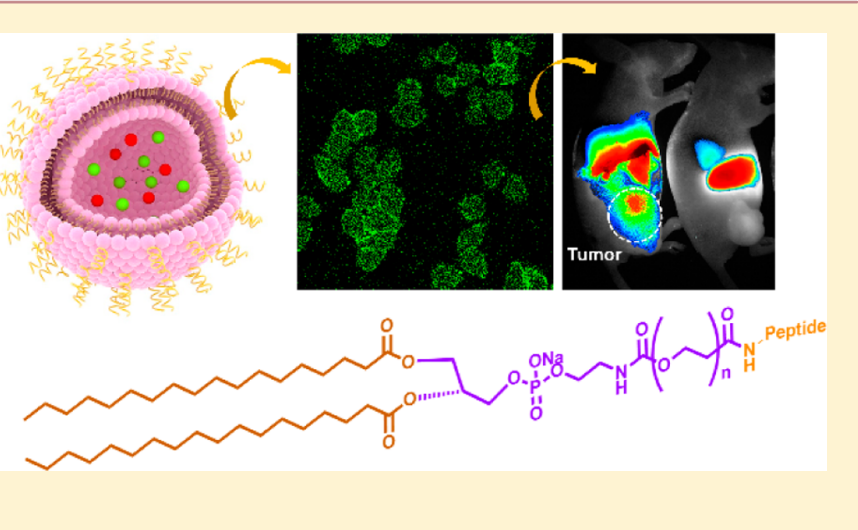

was the first targeting drug to obtain FDA approval for use in HER2 overexpressed breast cancer. ${ }^{18}$ Advancements in nanomaterial and nanotechnology have provided a promising strategy for cancer targeting drug delivery. ${ }^{19,20}$ Recent researches have shown that the drug delivery efficiency of nanocarriers could be dramatically enhanced by a modification of the specific recognition molecules. ${ }^{21}$ As promising interface molecules, peptides may be a good choice of recognition element to modify liposomes in order to increase the targeting specificity. ${ }^{22-26}$ For instance, one type of tumor metastasis targeting peptide was conjugated with the doxorubicin containing liposome to realize drug delivery. ${ }^{27}$ The delivery efficiency was enhanced by TAT (a penetrating peptide) modified liposomes ${ }^{28}$ and GE11 (a targeting peptide) modified liposomes to epidermal growth factor receptor (EGFR) positive nonsmall cell lung cancer. ${ }^{29}$

Herein, H6-modified liposomes (H6-LS) were prepared by thin film hydration method. We hypothesized that the H6modified nanocarrier may be a promising candidate for targeting delivery of antitumor drugs toward HER2 positive breast cancers. H6, a novel peptide (YLFFVFER) was proposed by high throughput library screening toward HER2 in our previous work. ${ }^{30}$ This octapeptide (YLFFVFER) showed nanomolar affinity toward HER2. H6 could be conjugated with amphiphilic molecular 1,2-distearoyl-sn-glycero-3-phosphoethanolamine- $N$-[poly(ethylene glycol)-2000]- $N$-hydroxy-

Received: January 19, 2016

Accepted: February 10, 2016

Published: February 10, 2016 
succinimidyl (DSPE-PEG 2000 -NHS) by the nucleophilic substitution reaction to obtain a targeting compound $\mathrm{H} 6-\mathrm{PEG}_{2000^{-}}$ DSPE. The reaction efficiency was improved by our micromixer. Furthermore, the specific nanoparticles modified by H6$\mathrm{PEG}_{2000}$-DSPE (H6-LS) showed a good affinity toward human breast cancer (SKBR3, overexpressing HER2) cell line. Antitumor drugs encapsulated in the liposomes were successfully delivered into tumor cells in vitro. Additionally, we further demonstrate that our nanocarrier loading drugs could inhibit the growth of HER2 overexpressing tumor in vivo. As we expected, this nanocarrier loading drug system show high efficiency of drug targeting delivery.

At the first stage, the micromixer was fabricated with two slices of bonded polished chrome glasses (the substrate and the cover) using the conventional lithography strategy. (The fabrication details were shown in Supporting Information.) Briefly, the designed microstructure was displaced to the chrome glass by undergoing a series of treatments as described in the literature, i.e., exposure, development, dechroming, and etching to get the substrate plate. The size of the micromixer chip is $75 \mathrm{~mm}(\mathrm{~L}) \times 25 \mathrm{~mm}(\mathrm{~W}) \times 2 \mathrm{~mm}(\mathrm{H})$. The chip mainly consists of a reaction chamber with the size of $600 \mu \mathrm{m}$ $(\mathrm{W}) \times 43 \mathrm{~mm}(\mathrm{~L})$, three inlets, and one outlet. Inside of the reaction chamber, an uneven structure was fabricated, and the about 20 bulges were formed in order to make the disturbance of the reaction liquid. Synthesis of the H6-PEG ${ }_{2000}-\mathrm{NHS}$ was carried out in the fabricated micromixer. H6 peptide (M.W. $1119 \mathrm{Da}$ ) was synthesized (Figure S1) and conjugated with 1,2distearoyl-sn-glycero-3-phosphoethanolamine- $N$-[poly(ethylene glycol)-2000]-N-hydroxysuccinimidyl (DSPE-PEG $2000-\mathrm{NHS}$ M.W. $3000 \mathrm{Da}$ ) in the micromixer chamber to obtain the targeting compound $\mathrm{H} 6-\mathrm{PEG}_{2000}-\mathrm{DSPE}$. As shown in Scheme 1a, peptide $\mathrm{H} 6$ dissolved in newly distilled $N, N$-dimethylfor-

Scheme 1. Schematic of Microfluidic Device and Liposomes: (a) Structure of the Micromixer and (b) Structure of H6 Modified Liposomes (Encapsulated with FITC and DOX)

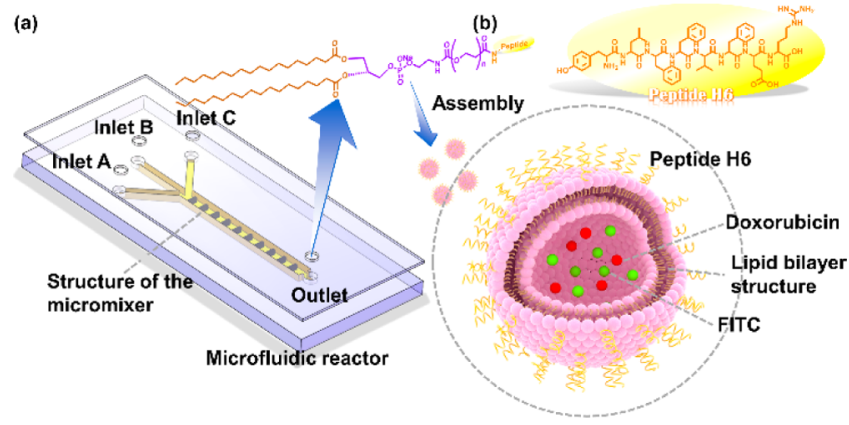

mamide (DMF) $(2 \mathrm{mg} / \mathrm{mL})$ was introduced from inlet $\mathrm{A}$, and DSPE-PEG ${ }_{2000}$-NHS in DMF $(3 \mathrm{mg} / \mathrm{mL})$ was introduced from inlet $\mathrm{C}$ at the same flow rate of $0.56 \mu \mathrm{L} / \mathrm{min}$. $N, N$ Diisopropylethylamine (DIPEA) in DMF (1:100) was introduced from inlet B to adjust the base condition. After 15 $\mathrm{h}$, targeting compound was collected from the outlet.

We used high-performance liquid chromatography (HPLC) to monitor the micromixer based reaction. As shown in Figure 1a, the peak of peptide was decreased and the peak of peptide$\mathrm{PEG}_{2000}$-DSPE was increased along the time. After $15 \mathrm{~h}$, the conversion rate was achieved $90 \%$. Compared to the conventional vessels (Figure S2), the micromixer reaction is low consumption and high efficiency. Then we used matrix-assisted

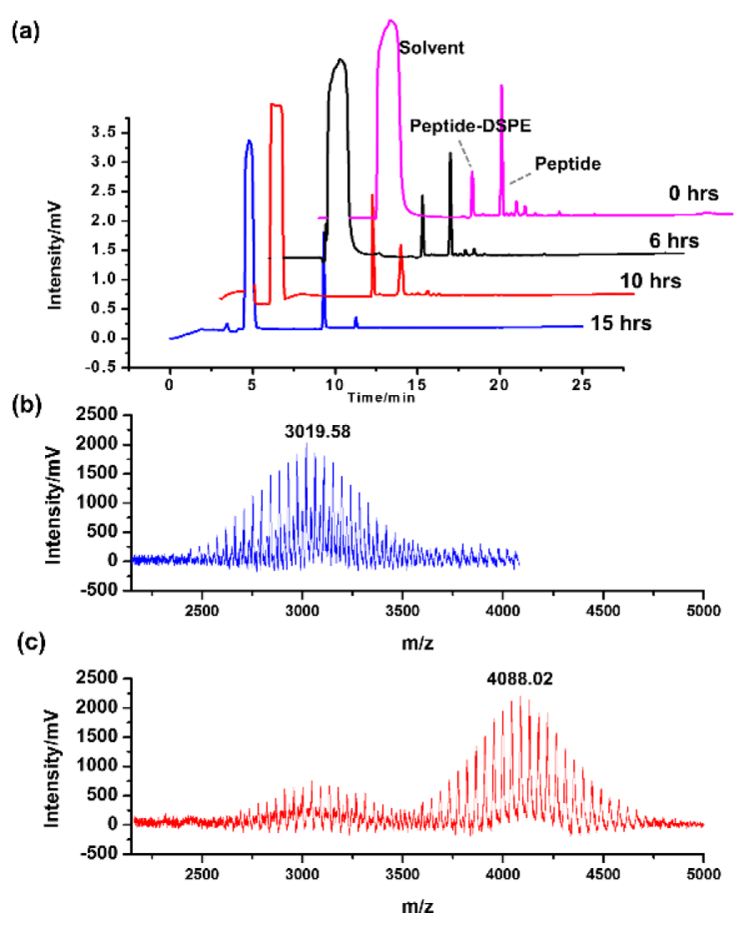

Figure 1. Monitoring of the conjugation between peptide and DSPE$\mathrm{PEG}_{2000}$-NHS. (a) HPLC spectra of reaction mixture at different times. (b) MALDI-TOF-MS spectra of NHS-PEG ${ }_{2000}$-DSPE. (c) MALDI-TOF MS spectra of $\mathrm{H}_{6}-\mathrm{PEG}_{2000}$-DSPE.

laser desorption ionization time of flight mass spectrometry (MALDI-TOF MS) to identify the products. The mass peak of the reactants 3019.58 (DSPE-PEG ${ }_{2000}$-NHS, Figure $1 \mathrm{~b}$ ) and the mass peak of $15 \mathrm{~h}$ product is 4088.02 (H6-PEG ${ }_{2000}$-DSPE, Figure 1c), indicating a successful and effective conjugation based on the micromixer.

Liposomes were then prepared by the conventional thin lipid film method (the preparation details were shown in Supporting Information). Briefly, soy phospholipids/cholesterol/H6$\mathrm{PEG}_{2000}$-DSPE (molar ratio 20:10:2) were dissolved in dichloromethane/methanol mixed dissolvent. The mixed solvent was evaporated under reduced pressure at $40{ }^{\circ} \mathrm{C}$ to form a lipid film, which was subsequently hydrated with phosphate-buffered saline (PBS) and sonicated for $30 \mathrm{~min}$ with a bath type sonicator to obtain liposomes. The morphologies of liposomes loading doxorubicin (LS-DOX) and H6-liposomens loading doxorubicin (H6-LS-DOX) were shown by TEM. Both LS-DOX and H6-LS-DOX were spherical in shape and had good dispersion (Figure 2). The average hydrodynamic diameter and surface charge were characterized by measuring the size and zeta potential with dynamic light scattering (DLS), as shown in Figure 2a,b. The diameter of LS-DOX was around $90 \mathrm{~nm}$, and the zeta potential in PBS was around $-14.0 \mathrm{mV}$, while the diameter of H6-LS-DOX was around $130 \mathrm{~nm}$ and the zeta potential was around $-20 \mathrm{mV}$. It is indicated that after modification both the size and systematic stability were increased. Furthermore, encapsulated efficiency was assessed. Both LS and H6-LS-DOX showed high encapsulation efficiencies (Figure S3). To investigate the DOX release in vitro, the required quantity of DOX-loaded liposomes was transferred into a dialysis bag. All of the release rates of DOX from loaded liposomes were much slower than that of DOX-sol (Figure S5). Then, the stability of DOX-loaded liposomes in serum was investigated using a filtration test. The fluorescence 
(a)

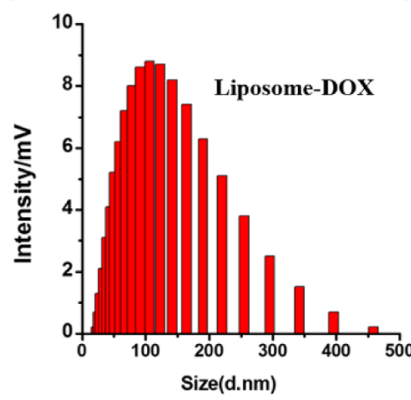

c)

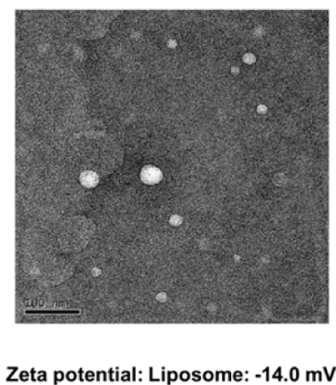

(b)

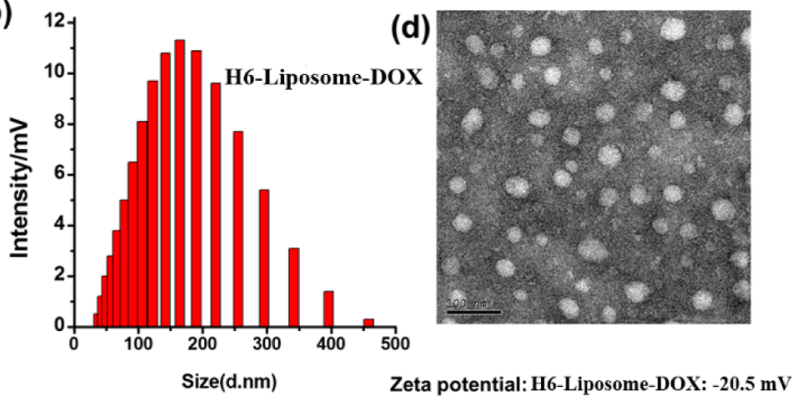

Figure 2. Characteristics of different liposomes. (a) Size distribution of unmodified liposomes loading doxorubicin. (b) Size distribution of DSPE-PEG ${ }_{2000}-\mathrm{H} 6$ modified liposomes loading doxorubicin. (c) Transmission electron microscopy (TEM) graph and zeta potential of unmodified liposomes loading doxorubicin. (d) TEM graph and zeta potential of DSPE-PEG 2000 -H6 modified liposomes loading doxorubicin.

of LS-DOX, SP-LS-DOX, and H6-LS-DOX did not show obvious changes after incubation in serum at $37{ }^{\circ} \mathrm{C}$ for $24 \mathrm{~h}$ followed by membrane filtration (Figure S4). In other words, most of liposomes appeared to retain their state without aggregation. These results indicated the substantial stability of DOX-loaded liposomes.

Then we continued to observe the bioactivity of the prepared liposomes. Confocal microscopy (Olympus FV1000-IX81 confocal-laser scanning microscope) was used to investigate cellular uptake of the liposomes (Figure 3). We employed FITC (fluorescein isothiocyanate) as the mimic of the hydrophilic drug. When lipid film was hydrated with phosphate-buffered saline (PBS), FITC could be loaded in liposomes. Human breast cancer cells SKBR3 (HER2 overexpression cancer cells) and Human Embryonic Kidney 293A (HER2 nonexpression cells) were applied as the positive cell and control cell models, respectively. SP (EFVYFLRF), a sequence scrambled peptide was served as negative control peptide. The above cells were incubated with H6-LS-FITC (15 $\mu \mathrm{g} / \mathrm{mL}, 200 \mu \mathrm{L})$, LS-FITC $(15 \mu \mathrm{g} / \mathrm{mL}, 200 \mu \mathrm{L})$, and SP $(15$ $\mu \mathrm{g} / \mathrm{mL}, 200 \mu \mathrm{L}$ ) as well as Hoechst 33342 (nucleus indicator, $10 \mu \mathrm{g} / \mathrm{mL})$. The confocal images (60×, oil-immersion objective) were obtained in the excitation wavelength of both $488 \mathrm{~nm}$ (FITC) and the $405 \mathrm{~nm}$ (Hoechst 33342). After 15 min of incubation, the SKBR3 cells treated with LS-FITC (liposome loading FITC) and SP-LS-FITC exhibited very low fluorescence intensity (Figure 3a,c). However, H6-LS-FITC demonstrated much more fluorescent intensity in SKBR3 Cells in Figure 3b, revealing the higher uptake in SKBR3 cell line. Again, very low fluorescence was observed in 293A cell lines in Figure 3d, revealing that the enhanced uptake of H6-LS could

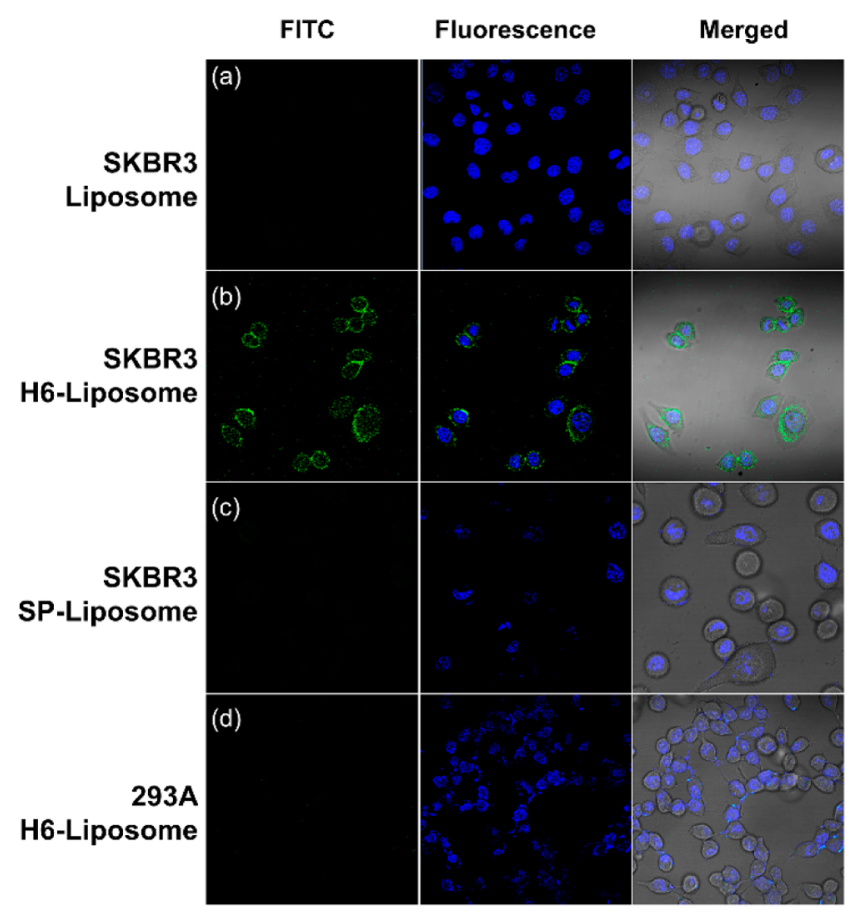

Figure 3. Confocal microscopy images of liposomes toward cells. Green fluorescence represents FITC, and blue fluorescence is for Hoechst 33342. (a) SKBR3 cells were incubated with LS-FITC. (b) SKBR3 cells were incubated with H6-LS-FITC. (c) SKBR3 cells were incubated with SP-LS-FITC. (d) 293A cells were incubated with H6LS-FITC.

be mediated by HER2, the H6 receptor, very specifically. These results have further proved that liposomes modified with $\mathrm{H} 6$ peptide have a remarkable specific recognition ability toward HER2 positive breast cancer cells.

We estimated that the endocytosis would be different between the H6-LS-FITC and LS-FITC. We designed the cell assays to monitor the endocytosis and release of both the liposomes along with time in different cell lines. SKBR3 were incubated with H6-LS-FITC $(15 \mu \mathrm{g} / \mathrm{mL}, 200 \mu \mathrm{L})$ and LSFITC $(15 \mu \mathrm{g} / \mathrm{mL}, 200 \mu \mathrm{L})$ as well as Hoechst $33342(10 \mu \mathrm{g} /$ $\mathrm{mL}$ ). Confocal image was also captured at the time point of 0 , 5, 10, and $15 \mathrm{~min}$, respectively. As show in Figure 4, after 5 min incubation, the green fluorescence of H6-LS-FITC has appeared with little fluorescence of LS-FITC (Figure 4a,b,e,f). When it came to $10 \mathrm{~min}$ incubation of H6-LS-FITC, fluorescence intensities on the SKBR3 cell membrane are getting stronger and showing the tendency to diffuse into cells (Figure 4g). After 15 min incubation, H6-LS-FITC has been endocytosed and released into the cytoplasm (Figure $4 \mathrm{~h}$ ). After the H6-LS-FITC incubation time of $20 \mathrm{~min}$ or longer, the situation is similar as the one of $15 \mathrm{~min}$. We estimated the endocytosis has achieved saturation at about $15 \mathrm{~min}$. However, after incubation with LS-FITC for $15 \mathrm{~min}$ a low green fluorescence appears on the cell membrane Figure $4 a-d$. Green fluorescence intensities in cells treated with H6-LS-FITC were significantly higher than those who exposed to LS-FITC at all test time. Obviously, the HER2 targeting peptide H6 plays a key role as the interface molecules between the biosystems and the liposomes. It demonstrates that $\mathrm{H} 6$ peptides could enhance tumor targeting and penetration of drug encapsulated nanocarriers and is a promising candidate for developing targeted drug delivery systems. 


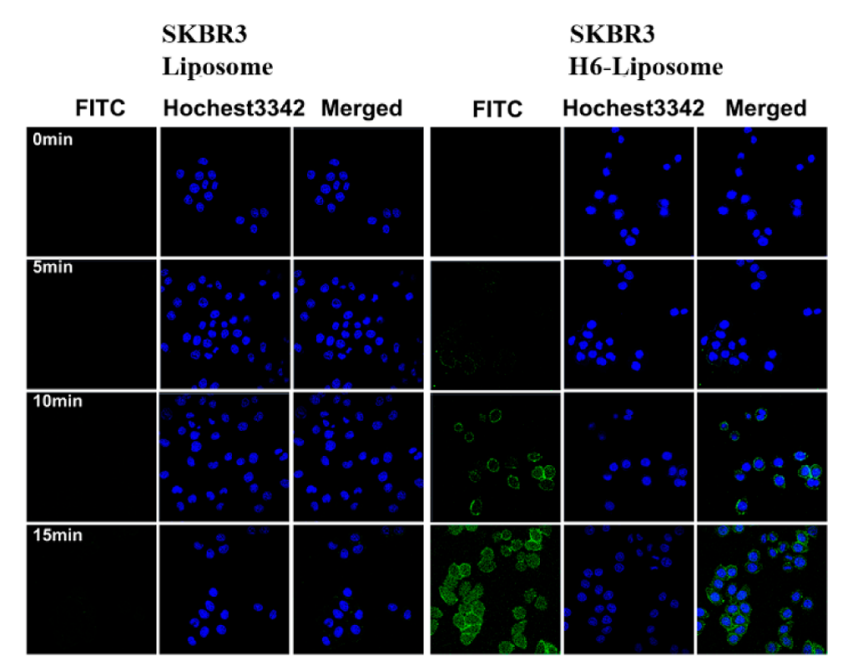

Figure 4. Fluorescence confocal images of SKBR3 incubated with LSFITC and H6-LS-FITC cellular uptake at different times.

Furthermore, we investigated the antitumor activity against SKBR3 cells in vitro with $\mathrm{H} 6$ modified liposomes in which DOX was encapsulated. LS-DOX, H6-LS-DOX, and SP-LSDOX with different DOX concentration of 0.001, 0.01, 0.1, 1, 10 , and $100 \mu \mathrm{g} / \mathrm{mL}(150 \mu \mathrm{L})$ incubated SKBR3, respectively. After $24 \mathrm{~h}$ treatment with LS-DOX, H6-LS-DOX, and SP-LSDOX, the cell viabilities were measured by 3-(4,5-dimethyl-2thiazolyl)-2,5-diphenyl-2H-tetrazolium bromide (MTT) assay. As show in Figure S7, at the DOX concentration of $0.01 \mu \mathrm{g} /$ $\mathrm{mL}$, the cell viability of SKBR3 is over $60 \%$, while H6-LS-DOX was lower than LS-DOX and SP-LS-DOX. When DOX concentrations turned to $100 \mu \mathrm{g} / \mathrm{mL}$, the cell viability of SKBR3 decreased to $20 \%$. Furthermore, the half maximal inhibitory concentration $\left(\mathrm{IC}_{50}\right)$ values at $24 \mathrm{~h}$ of H6-LS-DOX was 5.2-fold, 6.5-fold, and 17-fold lower than that of LS-DOX, SP-LS-DOX, and DOX. The deference may be attributed to the improved targeting efficacy of the H6-modified liposomes. As expected, H6-LS-DOX displayed a significantly greater efficacy relative to LS-DOX and SP-LS-DOX. In other words, H6-LSDOX was more effective to kill the HER2 overexpressed breast cancer cells than LS-DOX, which might be promising in specific delivery of therapy or imaging agents to breast cancers.

To examine whether the molecular delivery effects were viable in the in vivo conditions, we first performed optical imaging experiments in tumor xenograft model by using HER2overexpressed SKBR3 cells. 1,1-Dioctadecyl-3,3,3,3-tetramethylindotricarbonyaineiodide (DiR) was encapsulated in the liposomes to realize the in vivo imaging. Similar to the above, H6-LS-DiR and LS-DiR were both prepared and injected form the tail vein $(1 \mathrm{mg} / \mathrm{mL}, 200 \mu \mathrm{L})$ at a dose corresponding to 1 $\mu \mathrm{g} / \mathrm{mL}$ of DiR. Mice treated with PBS only were served as negative control. Figure $5 \mathrm{a}, \mathrm{b}$ shows the real-time biodistribution and tumor accumulation of the different liposomes at 4 and $8 \mathrm{~h}$ postinjection. After $4 \mathrm{~h}$, the tumor fluorescence intensity in H6-LS-DiR treated mice was slightly higher than LS-DiR. After $8 \mathrm{~h}, \mathrm{H} 6-\mathrm{LS}-\mathrm{DiR}$ showed a good targeting efficiency and accumulated continuously in tumor sites, while LS-DiR showed a relatively low fluorescence intensity. After 8 $h$, tumor tissues were excised and their fluorescence images were recorded. As shown in Figure 5c,d, the fluorescence levels in the livers of nanocarrials-treated mice were very high, which might be attributed to the high macrophage uptake nature of the liver. H6-LS-DiR showed higher tumor target ability and
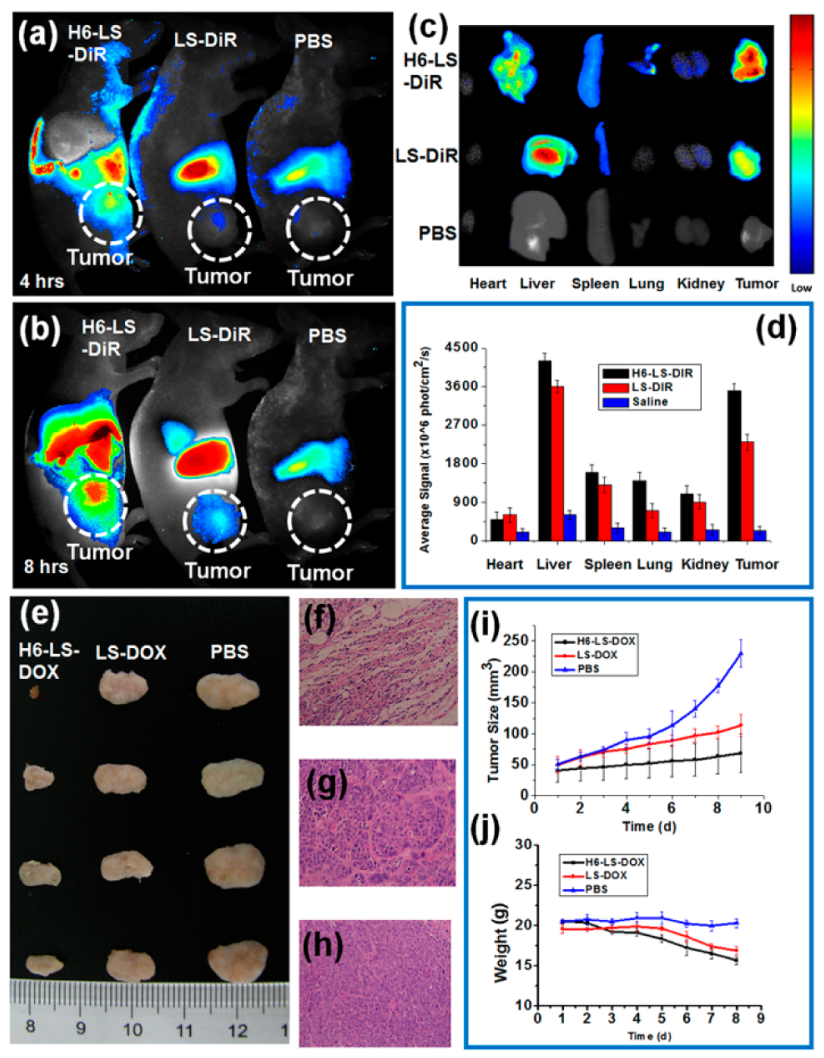

Figure 5. In vivo and ex vivo imaging of tumor targeting delivery by H6-LS-DiR and LS-DiR. (a,b) Real-time biodistribution and tumor accumulation of H6-LS-DiR, LS-DiR, and PBS treatment at 4 and $8 \mathrm{~h}$. (c) Ex vivo fluorescence imaging of tumor accumulation and biodistribution. (d) Quantification of the fluorescence signals ex vivo. Fluorescence intensity was measured in counts/energy/area and is presented as an average $(n=4)$. (e) Excised tumor photograph after LS aided drug delivery. $(\mathrm{f}-\mathrm{h}) \mathrm{H} \& \mathrm{E}$ stained excised tumor of the mice after H6-LS-DOX, LS-DOX, and PBS treatment. (i) The tumor size changing curves in the monitored 9 days. (j) The mice weight changing curves in the monitored 9 days.

the fluorescence intensity than LS-DiR, which indicated that the target peptide $\mathrm{H} 6$ plays an important role in the molecular delivery process.

Next we did in vivo drug delivery and cure assays. Tumor inhibition studies were carried out to examine the efficiency of H6-LS-DOX, LS-DOX, and saline. SKBR3 cells were also xenografted in nude mice to construct the tumor models. After the tumors had been allowed to develop approximately 50-120 $\mathrm{mm}^{3}$ the mice were randomly divided into three groups $(n=4)$ in order to minimize the random errors of the weights and tumor-sizes. H6-LS-DOX, LS-DOX, and PBS were intravenously injected into tumor-bearing mice via tail veins. The mice were treated with the nanocapsules $(300 \mu \mathrm{L})$ each day for six consecutive days at a dose corresponding to $100 \mu \mathrm{g} / \mathrm{mL}$ of DOX. The tumor sizes and the mice weights were monitored in the 6 days and in the following 3 days. Therefore, observation was carried out in 9 consecutive days. The results were determined and are shown in Figure $5 \mathrm{~d}-\mathrm{j}$. The tumors treated with H6-LS-DOX are much smaller than those treated with LSDOX and those untreated (Figure 5e,i), which indicated an efficient therapeutic effect. After cure assays, the tumor tissue sections were analyzed by $\mathrm{H} \& \mathrm{E}$ (hematoxylin-eosin) staining for evaluation of the morphology. Compared to the control group, H6-LS-DOX treatment caused the loose spaces between 
tumor tissues. The obvious changes of the tumor morphology indicated that the efficient tumor apoptosis (Figure $5 \mathrm{f}-\mathrm{h}$ ). The body weight of the mice treated with H6-LS-DOX, LS-DOX, and PBS were also shown in Figure 5j. It revealed that the nanocapsules have delivered the drugs to the tumor site in an effective way by the modification of $\mathrm{H} 6$ with low side effects.

H6-LS loaded drugs may be prospects for drug delivery. Because the high affinity of H6-LS and SKBR3 cells high inhibition of H6-LS-DOX, our nanocarrier drug delivery is promising in clinical targeting chemotherapy for killing human breast cancer. For further study, we ought to do more effort to realize our goal, applying our nanocarrier drug delivery to clinical therapy.

\section{ASSOCIATED CONTENT}

\section{S Supporting Information}

The Supporting Information is available free of charge on the ACS Publications website at DOI: 10.1021/acsmedchemlett.6b00028.

Some experimental details, encapsulation efficiency, inhibition studies, and some additional figures (PDF)

\section{AUTHOR INFORMATION}

\section{Corresponding Authors}

*E-mail (W.W): wangwz@nanoctr.cn.

*E-mail (Y.J.): jiayunhong2012@163.com.

*E-mail (Z.H.): huzy@nanoctr.cn.

\section{Author Contributions}

"These authors contributed equally to this work.

Notes

The authors declare no competing financial interest.

\section{ACKNOWLEDGMENTS}

We acknowledge funding from the National Natural Science Foundation of China (21305023, 31270875, and 31470049), Program for the Top Young Talents of Beijing (2015000021223ZK36), Beijing Municipal Natural Science Foundation (2144058) and National High Technology Research and Development Program of China (2015AA020408).

\section{REFERENCES}

(1) Siegel, R. L.; Miller, K. D.; Jemal, A. Cancer statistics, 2015. CaCancer J. Clin. 2015, 65, 5-29.

(2) Lu, B.; Xiong, S. B.; Yang, H.; Yin, X. D.; Chao, R. B. Solid lipid nanoparticles of mitoxantrone for local injection against breast cancer and its lymph node metastases. Eur. J. Pharm. Sci. 2006, 28, 86-95.

(3) Carney, W. P.; Leitzel, K.; Ali, S.; Neumann, R.; Lipton, A. HER2/neu diagnostics in breast cancer. Breast Cancer Res. 2007, 9, 207.

(4) Tai, W.; Mahato, R.; Cheng, K. The role of HER2 in cancer therapy and targeted drug delivery. J. Controlled Release 2010, 146, 264-275.

(5) Shi, J.; Votruba, A. R.; Farokhzad, O. C.; Langer, R. Nanotechnology in drug delivery and tissue engineering: from discovery to applications. Nano Lett. 2010, 10, 3223-30.

(6) Adair, J. H.; Parette, M. P.; Altınoğlu, E. İ.; Kester, M. Nanoparticulate Alternatives for Drug Delivery. ACS Nano 2010, 4, 4967-4970.

(7) Chang, M.; Lu, S.; Zhang, F.; Zuo, T.; Guan, Y.; Wei, T.; Shao, W.; Lin, G. RGD-modified $\mathrm{pH}$-sensitive liposomes for docetaxel tumor targeting. Colloids Surf. Colloids Surf., B 2015, 129, 175-82.

(8) Du, B.; Han, S.; Li, H.; Zhao, F.; Su, X.; Cao, X.; Zhang, Z. Multifunctional liposomes showing radiofrequency-triggered release and magnetic resonance imaging for tumor multi-mechanism therapy. Nanoscale 2015, 7, 5411-5426.

(9) Kausik, R.; Han, S. Dynamics and state of lipid bilayer-internal water unraveled with solution state $1 \mathrm{H}$ dynamic nuclear polarization. Phys. Chem. Chem. Phys. 2011, 13, 7732-46.

(10) Liu, J.; Ma, H.; Wei, T.; Liang, X. J. CO2 gas induced drug release from $\mathrm{pH}$-sensitive liposome to circumvent doxorubicin resistant cells. Chem. Commun. (Cambridge, U. K.) 2012, 48, 4869-71.

(11) Jeon, T. J.; Poulos, J. L.; Schmidt, J. J. Long-term storable and shippable lipid bilayer membrane platform. Lab Chip 2008, 8, 1742-4.

(12) Zhang, W.; Peng, F.; Zhou, T.; Huang, Y.; Zhang, L.; Ye, P.; Lu, M.; Yang, G.; Gai, Y.; Yang, T.; Ma, X.; Xiang, G. Targeted delivery of chemically modified anti-miR-221 to hepatocellular carcinoma with negatively charged liposomes. Int. J. Nanomed. 2015, 10, 4825-36.

(13) Tao, Y.; Han, J.; Dou, H. Brain-targeting gene delivery using a rabies virus glycoprotein peptide modulated hollow liposome: biobehavioral study. J. Mater. Chem. 2012, 22, 11808.

(14) Leung, K. [18F]Fluorodipalmitin-labeled liposomes. In Molecular Imaging and Contrast Agent Database (MICAD); National Center for Biotechnology Information (US): Bethesda, MD, 2004.

(15) Bae, K. H.; Chung, H. J.; Park, T. G. Nanomaterials for cancer therapy and imaging. Mol. Cells 2011, 31, 295-302.

(16) Eloy, J. O.; Claro de Souza, M.; Petrilli, R.; Barcellos, J. P.; Lee, R. J.; Marchetti, J. M. Liposomes as carriers of hydrophilic small molecule drugs: strategies to enhance encapsulation and delivery. Colloids Surf., B 2014, 123, 345-63.

(17) Shi, M.; Lu, J.; Shoichet, M. S. Organic nanoscale drug carriers coupled with ligands for targeted drug delivery in cancer. J. Mater. Chem. 2009, 19, 5485.

(18) Romond, E. H.; Perez, E. A.; Bryant, J.; Suman, V. J.; Geyer, C. E., Jr.; Davidson, N. E.; Tan-Chiu, E.; Martino, S.; Paik, S.; Kaufman, P. A.; Swain, S. M.; Pisansky, T. M.; Fehrenbacher, L.; Kutteh, L. A.; Vogel, V. G.; Visscher, D. W.; Yothers, G.; Jenkins, R. B.; Brown, A. M.; Dakhil, S. R.; Mamounas, E. P.; Lingle, W. L.; Klein, P. M.; Ingle, J. N.; Wolmark, N. Trastuzumab plus adjuvant chemotherapy for operable HER2-positive breast cancer. N. Engl. J. Med. 2005, 353, 1673-84.

(19) Egusquiaguirre, S. P.; Igartua, M.; Hernandez, R. M.; Pedraz, J. L. Nanoparticle delivery systems for cancer therapy: advances in clinical and preclinical research. Clin. Transl. Oncol. 2012, 14, 83-93.

(20) Raemdonck, K.; Braeckmans, K.; Demeester, J.; De Smedt, S. C. Merging the best of both worlds: hybrid lipid-enveloped matrix nanocomposites in drug delivery. Chem. Soc. Rev. 2014, 43, 444-72.

(21) Wang, T.; Zhen, Y.; Ma, X.; Wei, B.; Wang, N. Phospholipid bilayer-coated aluminum nanoparticles as an effective vaccine adjuvant-delivery system. ACS Appl. Mater. Interfaces 2015, 7, 6391-6.

(22) Messerschmidt, S. K.; Musyanovych, A.; Altvater, M.; Scheurich, P.; Pfizenmaier, K.; Landfester, K.; Kontermann, R. E. Targeted lipidcoated nanoparticles: delivery of tumor necrosis factor-functionalized particles to tumor cells. J. Controlled Release 2009, 137, 69-77.

(23) Lu, J.; Jeon, E.; Lee, B. S.; Onyuksel, H.; Wang, Z. J. Targeted drug delivery crossing cytoplasmic membranes of intended cells via ligand-grafted sterically stabilized liposomes. J. Controlled Release 2006, $110,505-13$.

(24) Levy, R.; Thanh, N. T.; Doty, R. C.; Hussain, I.; Nichols, R. J.; Schiffrin, D. J.; Brust, M.; Fernig, D. G. Rational and combinatorial design of peptide capping ligands for gold nanoparticles. J. Am. Chem. Soc. 2004, 126, 10076-84.

(25) Lee, D.; Zhao, J.; Yang, H.; Xu, S.; Kim, H.; Pacheco, S.; Keshavjee, S.; Liu, M. Effective delivery of a rationally designed intracellular peptide drug with gold nanoparticle-peptide hybrids. Nanoscale 2015, 7, 12356-12360.

(26) Patra, S.; Roy, E.; Karfa, P.; Kumar, S.; Madhuri, R.; Sharma, P. K. Dual-responsive polymer coated superparamagnetic nanoparticle for targeted drug delivery and hyperthermia treatment. ACS Appl. Mater. Interfaces 2015, 7, 9235-46.

(27) Wang, Z.; Yu, Y.; Dai, W.; Lu, J.; Cui, J.; Wu, H.; Yuan, L.; Zhang, H.; Wang, X.; Wang, J.; Zhang, X.; Zhang, Q. The use of a 
tumor metastasis targeting peptide to deliver doxorubicin-containing liposomes to highly metastatic cancer. Biomaterials 2012, 33, 8451-60. (28) Qin, Y.; Chen, H.; Yuan, W.; Kuai, R.; Zhang, Q.; Xie, F.; Zhang, L.; Zhang, Z.; Liu, J.; He, Q. Liposome formulated with TATmodified cholesterol for enhancing the brain delivery. Int. J. Pharm. 2011, 419, 85-95.

(29) Cheng, L.; Huang, F. Z.; Cheng, L. F.; Zhu, Y. Q.; Hu, Q.; Li, L.; Wei, L.; Chen, D. W. GE11-modified liposomes for non-small cell lung cancer targeting: preparation, ex vitro and in vivo evaluation. Int. J. Nanomed. 2014, 9, 921-35.

(30) Wang, Z.; Wang, W.; Bu, X.; Wei, Z.; Geng, L.; Wu, Y.; Dong, C.; Li, L.; Zhang, D.; Yang, S.; Wang, F.; Lausted, C.; Hood, L.; Hu, Z. Microarray based screening of peptide nano probes for HER2 positive tumor. Anal. Chem. 2015, 87, 8367-72. 